Revue européenne des sciences sociales

European Journal of Social Sciences

XLI-125 | 2003

Index de la Revue européenne des sciences sociales

(1995-2002)

\title{
Autour de l'édition des œuvres de Pareto et sur ses interprétations
}

\section{Giovanni Busino}

\section{CpenEdition}

Journals

Édition électronique

URL : http://journals.openedition.org/ress/559

DOI : $10.4000 /$ ress. 559

ISSN : 1663-4446

Éditeur

Librairie Droz

Édition imprimée

Date de publication : 1 janvier 2003

Pagination : $55-75$

ISBN : 2-600-00844-6

ISSN : 0048-8046

Référence électronique

Giovanni Busino, "Autour de l'édition des œuvres de Pareto et sur ses interprétations », Revue

européenne des sciences sociales [En ligne], XLI-125 | 2003, mis en ligne le 01 décembre 2009, consulté le 01 mai 2019. URL : http://journals.openedition.org/ress/559 ; DOl : 10.4000/ress.559 


\section{Giovanni BUSINO}

\section{AUTOUR DE L'ÉDITION DES GEUVRES DE PARETO ET SUR SES INTERPRÉTATIONS}

Avec la publication d'un nouveau volume des «TEuvres complètes», le programme que nous nous étions fixé est désormais en voie d'achèvement. Ainsi la totalité des écrits parétiens est désormais à la disposition des lecteurs. Il est probable que d'autres textes seront portés à la lumière au fil des années prochaines. Cependant, l'essentiel de la production scientifique et littéraire de Pareto est désormais disponible. Les chercheurs disposent de tous les matériaux pour reconstituer la formation des doctrines parétiennes et pour en évaluer l'importance.

Les écrits et la correspondance montrent le libéral au combat, toujours méfiant à l'égard de tout ce qui porte atteinte à la liberté d'agir et de penser, très attaché à toutes les formes de la liberté d'expression, soucieux de transparence dans la vie publique, en quête d'une organisation économico-sociale décentrée, capable d'initiatives, laissant aux individus la responsabilité de leurs entreprises. Pas de dogmes ou de préjugés doctrinaires à ce propos. Entre la nationalisation et la privatisation d'entreprises à vocation nationale, telles les compagnies des chemins de fer, Pareto considère que la solution la plus efficace serait leur transformation en coopératives confiées aux soins des cheminots, c'est-à-dire aux travailleurs eux-mêmes. Il prône également la solution mutualiste pour les assurances sociales et le petit crédit. Les régulations sociales produites par le marché ne lui paraissent exiger qu'une seule condition: celle selon laquelle les acteurs puissent agir en conformité aux principes du bien commun et de l'intérêt général propres à chaque communauté humaine. L'économie n'explique pas tout, elle ne justifie pas non plus tout. Pareto sait que le champ social la déborde très largement. D'où l'impératif pour tous de faire constamment preuve de prudence et de pondération.

L'extrême et changeante complexité des actions humaines pousse le libéral Pareto à être souvent insolent même vis-à-vis des doctrines qui sont les siennes. N'est-ce pas là la preuve de son libéralisme authentique? Certes, il considère la démocratie le moindre mal possible. Et pour cela il fait continuellement appel au bon sens, à la raison, à la prudence, tout en rappelant qu'il n'y a pas de société sans la menace de la force, sans la détermination de l'utiliser lorsqu'il est indispensable de le faire. Ceux qui affirment le contraire, lui paraissent prêts à devenir des esclaves.

Cette revendication de la liberté et cette certitude que la force seulement peut garantir la survie des conditions d'exercice de la liberté, confèrent à la pensée de Pareto l'ambivalence et l'ambiguïté à l'origine des mouvements de rejet manifestés surtout par certains sociologues à son endroit. Et pourtant une lecture sereine, distante, critique, de ses écrits, nous montre que Pareto est, sans contestation possible, un classique des sciences sociales, l'un des pères fondateurs des 
sciences sociales modernes. À ce titre, ses livres peuvent nous aider dans notre travail d'aujourd'hui. C'est pour cette raison qu'on a éditée ses travaux et ses lettres.

Tous les écrits de Pareto rédigés en français, en anglais, en allemand et en espagnol se retrouvent, en effet, dans les volumes édités par Droz. Il est vrai que les études rédigées directement en italien figurent dans notre édition dans cette langue et que des raisons financières nous ont empêché de les faire traduire et publier en français. Il n'en reste pas moins que ces écrits en italien demeurent ainsi également accessibles.

On peut donc affirmer qu'à quatre-vingts ans de sa mort, « tout Pareto » a été réuni et republié: les œuvres principales, les écrits mineurs, mais aussi une partie importante de sa correspondance scientifique et personnelle. Cela a-t-il permis de formuler un jugement plus serein sur cet auteur «maudit» de la tradition sociologique?

Les études de Raymond Aron, Julien Freund, de Raymond Boudon, de Bernard Valade, d'Alban Bouvier, de Jean-Claude Passeron, de Philippe Steiner et de Marc Barbut, en France, sont là pour nous prouver que quelque chose a changé dans l'herméneutique parétienne depuis les temps de Célestin Bouglé, Maurice Halbwachs et Georges Gurvitch, où le mépris et l'indifférence étaient seuls de mise. Aujourd'hui, malgré les errements de Guy Perrin et l'ostracisme de la sociologie universitaire française, il y a quelques raisons d'espérer. Peut-être Raymond Aron a-t-il vu juste, en affirmant que «l'auteur du Traité ne jouira jamais, sur cette terre, d'une reconnaissance unanime et pacifique», mais enfin, il y a une bonne place pour ceux qui veulent connaître et juger plutôt que psalmodier ou (et) injurier.

Nul doute que les «Euvres complètes » constituent un moment important pour prendre la mesure de la pensée de Pareto et cela «dans le cadre du développement de la sociologie et de sa constitution en discipline rigoureuse» (J.Freund); nul doute que les «Euvres complètes » sont à l'origine d'un «revival » parétien d'une ampleur considérable et dont les bibliographies internationales des sciences sociales marquent l'ampleur et les rythmes annuels. Les quelques deux cents et plus articles consacrés aux «CEuvres complètes » par les recenseurs, ne nous font pourtant pas oublier que notre édition est un outil assez rudimentaire. Pas d'annotations, pas de variantes, pas d'appareils critiques: dans les volumes des «EEuvres complètes », il n'y a rien qui puisse faire de notre édition un monument semblable à «The Works and Correspondance of David Ricardo» de Piero Sraffa, ou bien aux «Collected Works of John Stuart Mill» de F.E.L. Priestley, ou même aux «Opere complete di Francesco Ferrara» de Federico Caffé. Mon édition n'est rien d'autre qu'un travail préliminaire, une sorte de reconnaissance dans la jungle des écrits parétiens, qui ne doit guère dispenser d'envisager, à plus ou moins courte échéance, la préparation d'une édition critique des œuvres et de la correspondance du sociologue de Céligny.

Pourquoi ai-je dû faire une édition préparatoire? Pourquoi n'ai-je pas entrepris d'emblée l'édition critique, comme certains continuent à me le reprocher durement, avec moins de virulence, aujourd'hui, il est vrai, qu'il y a un peu plus de quarante ans? Pourquoi je n'ai pas respecté l'ordre chronologique? Pourquoi certains articles sont-ils publiés plusieurs fois, en des versions différentes?

Puisque diverses personnes et quelques revues scientifiques me posent à nouveau ces mêmes questions, il faut bien que je me décide à y répondre clairement. Et ma réponse sera simple: aucune institution, aucune université, aucun 
mécène n'a voulu nous aider. Les difficultés et les obstacles auraient dû nous décourager et nous faire renoncer; ils nous ont seulement fait changer d'objectifs et de temps. Et voici très brièvement cette histoire, «notre histoire ».

Un concours de circonstances fortuit m'avait amené, voici plus de quarante ans, à étudier Pareto sans que je l'eusse désiré ni même voulu. Au contraire, ma formation personnelle et mes choix philosophiques et politiques m'en auraient plutôt tenu éloigné. Alors, comment cela se fit-il?

Avant les années 1955-1956, je savais bien peu de choses de l'économie et de la sociologie parétiennes. J'en avais, bien sûr, entendu parler par Jean Piaget, dans ses leçons d'histoire de la pensée scientifique et d'introduction à l'épistémologie génétique; j'avais lu, évidemment, ce que Pitirim Sorokin et Talcott Parsons en avaient écrit, mais je n'en savais guère plus. Une tentative de lire le Traité de sociologie générale avait lamentablement échoué: l'ennui et l'indifférence avaient eu raison de ma curiosité et de ma bonne volonté avant même que j'atteigne le paragraphe soixante-dix.

En 1958, je fus appelé à collaborer avec une équipe d'économistes, démographes et psychologues sociaux dans le cadre d'une recherche de grande envergure sur les conséquences sociales de l'industrialisation dans les pays du subcontinent asiatique. En étudiant le rôle et les fonctions des agents du changement, je rencontrais à nouveau Pareto le jour où, ayant à discuter la célèbre thèse de Schumpeter sur l'entrepreneur, je voulus en savoir davantage sur les élites sociales...

Cette fois-ci, le contact avec le Traité... fut moins désastreux. Je lus en effet les paragraphes qui m'intéressaient, tout en parcourant rapidement le reste. Sans être vraiment fasciné, j'eus tout de même envie de lire les écrits économiques de Pareto. Ce que je fis, dans la belle et confortable bibliothèque du club des officiers d'une merveilleuse ville frontalière du Pakistan occidental, en proie à des sentiments d'irritation et de lassitude.

Rentré en Suisse - car la recherche sur les conséquences sociales de l'industrialisation s'était peu à peu transformée en une enquête de marché sur les besoins sanitaires des populations étudiées - le professeur Jean-Charles Biaudet, alors président de l'Ecole de sciences sociales et politiques de l'Université de Lausanne, m'engagea, probablement à cause de ma connaissance de la langue italienne, à rassembler et à mettre un peu d'ordre dans les documents de Pareto qui étaient dispersés, pour ne pas dire éparpillés, en Suisse romande. Sans trop d'enthousiasme, et peut-être seulement par dette d'amitié à l'égard du professeur Biaudet, j'acceptais l'offre. Et c'est ainsi que débuta mon activité, ou aventure si on préfère, parétienne, menée ascétiquement, c'est-à-dire sans moyens ni aides d'aucune sorte, et dans les intervalles de temps libre que mes occupations principales me ménageaient de temps à autre.

Entré tardivement dans le microcosme parétien, exempt de passions et dépourvu d'intérêts, je fus très tôt impressionné par ce qu'il s'y passait et ce qu'il s'y faisait. Il existait alors, sur le plan international, une véritable secte parétienne, avec ses rites et ses liturgies, avec ses cardinaux, avec son service d'assistance, et même sa banque (le «Pareto Fund» de New York), très peu préoccupé par ce qu'il se passait ailleurs ou dans le voisinage.

Ma première réaction fut d'observer attentivement les activités des parétologues, tout en restant un laïc. Ni adepte ni compagnon de voyage, mais simple 
observateur. Etudier Pareto devait donc être un expédient pour étudier l'histoire des sciences sociales, en Suisse et en Europe; pour redécouvrir des filons de vie intellectuelle et éthico-politique justement, à mon avis, négligés dans la péninsule italienne et dans les pays francophones.

Parti de ce présupposé, il était évident que tous les écrits récents sur Pareto (sauf les exceptions d'usage) me laissaient dans l'ensemble perplexe: ceux de parétologues parce que hagiographiques, acritiques, peu sensibles aux implications culturelles et aux problématiques actuelles; ceux des autres, qui n'avaient pas lu, ou mal lu Pareto, et qui se prévalaient des thèses parétiennes pour consolider telle ou telle autre foi, défendre telle ou telle autre perspective idéologique, pour prôner ou pour discréditer, selon un point de vue partisan, une théorie, une doctrine, ou un système théorique. Dans les deux cas, il me semblait que la rigueur philologique n'était pas respectée.

Une chose, en effet, me frappa plus que tout autre: la lecture des textes garantie par des éditions philologiquement correctes, conditions généralement assurées pour la plupart des pères fondateurs des sciences sociales, se révélait impossible pour Pareto. La majeure partie des textes de cet auteur était introuvable, et ceux qui étaient disponibles s'avéraient inutilisables, expurgés ou adultérés. La première opération consistait donc en un sérieux redressement dans la meilleure tradition philologique: la première aune à laquelle on aurait mesuré les travaux ressortait de la philologie.

Mes premiers écrits parétiens reflétèrent, peut-être trop brutalement, tout cela; je dirais même qu'ils le réflèterent sans générosité ni équanimité à l'égard des travaux accomplis par mes prédécesseurs.

L'exemple le plus paradigmatique de ce que j'ai écrit plus haut me semble fourni par la revue critique des travaux sur Pareto, et plus particulièrement celle qui est consacrée aux études parues entre 1960 et 1965, aujourd'hui dans le volume Gli studi su Vilfredo Pareto oggi. Dall' agiografia alla critica, 1923-1973 (Roma, Bulzoni, 1974).

Dès qu'elle parut, de vives réactions fusèrent de toutes parts. Les parétiens m'accusèrent de déloyauté, de sauvagerie (oui, vraiment, de sauvagerie) et d'ignorance. On me taxa d'exhibitionnisme, de trahison, etc., etc.; on alla jusqu'à dire et à imprimer dans des revues célèbres parmi les initiés que j'avais voulu tenter un «coup de main», dans le but de m'approprier de l' «autorité d'exégèse » et de monopoliser en faveur d'une institution helvétique le droit d'auteur sur les œuvres parétiennes. Giuseppe Prezzolini dans son Manifesto dei conservatori (Milano, Rusconi, 1972, p.80) écrivit perfidement: «Oggi lo studio del Pareto è diventato un' industria, secondo quando leggo in uno scritto del Busino, che ne è certamente il più grande depositario e commerciante.»

D'un seul coup, stupéfait, je me trouvai au centre d'une furieuse et dans le fond ridicule polémique, qui eut même des prolongements comico-grotesques dans les colonnes d'un quotidien nippon à grand tirage.

Un article, peut-être le plus violent, certainement le moins empreint d'urbanité, paru dans une revue romaine à très faible diffusion, fut généreusement distribué par son auteur - par ailleurs chercheur talentueux - dans les milieux académiques suisses dans l'espoir de dénoncer «l'impie» et peut-être de bloquer son appel à l'Université de Lausanne. 
La connaissance limitée que l'on a chez les «social scientists» de la langue italienne, le soupçon que l'on nourrit ici à l'égard des publications d'inconnus, rendirent l'opération parfaitement gratuite. Toutefois, ce fut le signe le plus voyant de l'éclatement définitif de la secte parétienne. Dès lors, aucune collaboration, aucune coordination des études et des éditions n'étaient plus possibles. Du moins jusqu'à ce que les susceptibilités personnelles et les passions se seraient apaisées. Le comité international qui aurait dû éditer l'Opera omnia de Pareto, sur le modèle de l'édition française des œuvres d'Alexis de Tocqueville, sombra de son propre gré avant même de s'être valablement constitué.

Á la fin des années '50, j'élaborai un plan pour l'établissement d'une édition critique des œuvres de Pareto, sur le modèle des éditions de Ricardo et de Tocqueville. Les personnalités sollicitées m'éconduisirent plus ou moins aimablement. Tel président d'un fonds national pour la recherche scientifique me dit: «Pourquoi gaspiller des énergies et des moyens pour un auteur somme toute secondaire dans l'histoire des sciences sociales et dont seulement les Italiens devraient s'occuper?». Un professeur très influent, directeur d'un célèbre et richissime institut universitaire, me confia que pour lutter contre le communisme, il fallait s'occuper plutôt des auteurs socialistes et pas du tout des penseurs libéraux. Le seul à m'encourager chaleureusement dans mon entreprise fut Raffaele Mattioli, alors président de l'Institut italien pour les études historiques et président d'une importante banque d'affaires de la péninsule. C'est lui qui me présenta et me recommanda aux dirigeants d'une célèbre fondation culturelle américaine, lesquels me soumirent à un véritable examen et à une série d'épreuves très astreignantes. Après quatre séances plénières vraiment éprouvantes en présence d'un comité scientifique de ladite fondation, et au cours desquelles nous envisageâmes également mon transfert dans une université américaine, une décision très favorable fut prise. La Fondation décida de financer les travaux pour l'édition critique des œuvres de Pareto. Ce que je n'avais pas pu obtenir en Europe venait d'être décidé en quelques jours, à la fin d'un mois de février très froid, par des savants américains qui n'avaient pas manqué de m'éblouir par leurs exceptionnelles connaissances de la pensée sociale et du savoir sociologique du vieux continent. En vue de l'édition critique des œuvres de Pareto, cette importante fondation culturelle m'affecta donc une somme très élevée (400.000 dollars), destinée à m'attacher la collaboration d'un mathématicien, d'un économiste, d'un sociologue et d'un secrétaire-documentaliste pendant plusieurs années. La Fondation posait une seule condition: qu'une maison d'édition s'engageât à publier, en une édition impeccable, les manuscrits en l'espace d'une année, à dater du bon à tirer qui serait donné par un comité scientifique international composé de deux Suisses, deux Italiens, deux Français, un Allemand, trois Américains et un Japonais.

Des nombreux éditeurs furent contactés par Raffaele Mattioli et par moimême en Italie, en France, en Suisse, en Hollande, en Angleterre, et bien qu'il fût clair dès le début que les manuscrits auraient été livrés sans aucune obligation de verser des droits d'auteur, aucune maison d'édition ne jugea l'opération commercialement rentable. Toutes, hélas, refusèrent et pas toujours courtoisement.

Sur ces faits, des polémiques âpres au sujet des fondements de la pensée politique et sociale m'opposèrent, à plusieurs reprises, à des universitaires puissants et à des cardinaux sourcilleux de l'orthodoxie parétienne. Dès lors, des inimitiés féroces surgirent et les manigances pour nous rendre mutuellement la vie 
impossible ne se comptèrent plus. Le comité scientifique pour l'édition critique ne put par conséquent jamais se constituer valablement... Raffaele Mattioli, devant tant de difficultés, me conseilla de mettre en quarantaine le projet, mais de publier de temps en temps quelques œuvres épuisées ou introuvables, tout en attendant patiemment l'assoupissement des passions. Quelques mois plus tard, la grande Fondation américaine, ayant constaté mes déboires, eut la grâce d'annoncer à Mattioli la péremption de ses engagements financiers.

Tant d'années après, je peux avouer sans trop d'embarras avoir vécu ces refus comme une déconfiture et comme un défi personnel à relever d'une manière ou d'une autre. Et c'est dans cet état d'âme que j'ai alors commencé à envisager la possibilité de publier seul et à ma guise l'édition des œuvres complètes de Vilfredo Pareto, afin de prouver qu'une seule personne têtue par tempérament pouvait faire ce qu'il fallait. Rétrospectivement, j'affirme que la décision était déraisonnable, velléitaire, téméraire, et même plus qu'aventureuse. Je n'avais ni les compétences scientifiques, ni l'expérience du travail d'édition (j'avais à l'époque moins de 30 ans), ni les moyens matériels pour mener à bon port une entreprise si compliquée et complexe. Dois-je ajouter qu'en ces années-là je n'avais aucune sympathie pour l'œuvre de mon auteur, réputée plus ou moins réactionnaire, fortement méprisé par la corporation des sociologues à laquelle, bien que dans une position très critique, voire marginale, je considérasse appartenir? La particularité de ma position académique et scientifique faisait que je ne pouvais compter sur des collègues ou des maîtres pour me guider, me conseiller, pour évaluer ce que j'allais entreprendre.

Un coup de chance transforma mon projet en réalité. Ce coup de chance fut l'accession, en 1962, d'un vieil ami, Alain Dufour, chartiste-paléographe, à la direction générale des Editions Droz de Genève. Or, à la fin de cette même année, l'ancien procureur général du Canton de Vaud, Pierre Boven, consentit à me faire prendre connaissance d'un curieux dossier, provenant des archives du libraire lausannois François Rouge, dans lequel se trouvait un plan de réédition de certains écrits de Pareto. D'après les documents contenus dans ce dossier, on avait réussi, en février 1923, à persuader Pareto de réimprimer, à titre documentaire et par le procédé Darel, tout ce qu'il avait réédité en français, et ceci en suivant un plan de regroupement élaboré par Pasquale Boninsegni et Vladimiro Furlan. Pareto, selon Pierre Boven, avait donné, après bien des hésitations, son accord de principe, mais sous réserve de l'autorisation des Editions Payot, éditeur du Traité de sociologie générale, et celle du traducteur auquel l'auteur avait cédé les droits pour la version française. L'accord de Payot ne vint jamais. Ce même été, Pareto mourut et Rouge et les autres oublièrent aussitôt le projet. Le sociologue et économiste lausannois, malgré les efforts méritoires de G.-H. Bousquet, entra alors au purgatoire, là où, après leur mort, les auteurs de talent vont séjourner pendant quelques décennies.

Il faut rappeler qu'au début des années ' 60 , on pouvait se procurer le Manuel d'économie politique dans la version française et dans celle en langue castillane, ainsi que le Traité de sociologie générale dans la traduction anglaise d'Arthur Livingston et d'Andrew Bongiorno. Le marché de l'édition italienne, quant à lui, était mieux approvisionné. On trouvait en effet dans les bonnes librairies de la péninsule une excellente traduction italienne du Cours d'économie politique donnée par Renzo Fubini, un recueil exhaustif des écrits de théorie économique publiés par Giovanni Demaria, la version italienne des Systèmes socialistes par 
G.-H. Bousquet et Celestino Arena, le Traité de sociologie générale édité par Norberto Bobbio, de même que le petit ouvrage La transformation de la démocratie. Néanmoins, même en Italie les autres écrits ou textes importants de Pareto demeuraient introuvables. Ceux qui étaient disponibles s'avéraient peu fiables, étant expurgés ou adultérés. La règle philologique de la fidélité absolue au texte original, c'est-à-dire le dernier qui fût approuvé par l'auteur, se trouvait assez régulièrement violée. L'exemple le plus frappant est celui de l'édition Bousquet de Les systèmes socialistes, publiés tout d'abord à Paris en 1926, puis à Turin en 1951 et 1963. Cette édition comportait de nombreuses corrections au texte, plusieurs interversions de paragraphes, des changements dans l'agencement primitif de certaines phrases, des modifications de ponctuation et également des rectifications et unifications de citations et du système des références. En intervenant aussi massivement sur le texte, Bousquet était convaincu d'en rendre la lecture plus aisée, ou moins ardue. Mais en définitive, le seul résultat tangible aura été de mettre en circulation une série d'écrits apocryphes.

Mon projet d'éditer les CEuvres complètes de Vilfredo Pareto visait, en conséquence, à rendre accessible tous les écrits du professeur de Lausanne, et ceci dans une édition fiable, mais également à faire travailler ensemble des historiens, des sociologues et des économistes intéressés à l'histoire des sciences sociales. Dès lors, il fallait revenir aux textes originaux et les imprimer de la façon la plus fidèle possible, mais dans quel ordre? Fallait-il donner les textes dans l'ordre chronologique de leur parution ou de leur rédaction, ou bien suivant l'ordre thématique, ou systématique?

D'emblée, les partisans des différents points de vue se sont heurtés de front. Les "littéraires" voulaient l'édition chronologique tandis que les "scientifiques" réclamaient l'édition systématique délestée des écrits de circonstance ou trop en rapport avec l'actualité politique. Les premiers jugeaient que seul l'ordre chronologique permet de suivre le développement de la pensée d'un auteur, de comprendre ses mutations et d'en évaluer les enrichissements. Les autres faisaient valoir que l'actuelle division du travail scientifique oblige à départager les écrits selon qu'ils relèvent de l'économie, de la sociologie ou de la science politique. Les mêmes ajoutaient qu'il n'était pas indispensable de publier des écrits étrangers aux débats théoriques d'aujourd'hui; puisqu'ils n'avaient qu'une valeur purement historique, on pouvait toujours les consulter dans les bibliothèques et s'en servir pour les travaux d'érudition ou d'histoire de la pensée.

Par ailleurs, la tradition académique en la matière ne nous était d'aucun secours. En effet, on trouvait d'une part ceux qui avaient suivi l'optique chronologique et de l'autre ceux qui avaient choisi l'optique systématique. Prenons le cas classique des écrits de Turgot: l'édition de Dupont de Nemours (Belin \& Delance, 1808-1811, 9 tomes) est strictement chronologique; la deuxième d'Eugène Daire et d'Hyppolite Dussard (Guillaumin, 1844, 2 tomes) est systématique, alors que la troisième, celle de Gustave Schelle (Alcan, 1913-1923, 5 tomes) respecte la chronologie des parutions.

Les avantages d'un système par rapport à l'autre sont loin d'être évidents; en revanche, leurs inconvénients respectifs abondent et le fin connaisseur qu'était Luigi Einaudi en a dressé la liste. Il est certain que l'ordre chronologique n'est presque jamais intégralement respecté. Lorsqu'un auteur réunit en un volume des articles ou des mémoires parus à des époques différentes, faut-il republier les 
textes suivant la chronologie de leur parution ou les placer tous à la date de publication du recueil ? Là aussi, il n'y a pas de tradition bien arrêtée. Tantôt on répartit les textes dans la série chronologique, tantôt on en fait une entité autonome et on la place ensuite à la date de sa dernière parution. Einaudi, très favorable à l'ordre chronologique, considérait que le recueil élaboré par un auteur et paru de son vivant, possède une individualité historique spécifique qu'il faut sauvegarder. Il doit donc être inséré à la place chronologique qui est la sienne. Et que faire lorsqu'un même texte comporte différentes versions en plusieurs langues? Donner exclusivement la dernière parue du vivant de l'auteur, même si elle ne semble pas la meilleure et la plus complète? Les suppressions, les modifications, les changements doivent-ils être présentés comme des variantes, bien que la volonté ultime de l'auteur soit représentée par le dernier texte?

Pour un auteur comme Vilfredo Pareto, assez indiscipliné en fait de citations et de bibliographie, fallait-il rétablir et expliciter toutes les références? Fallait-il corriger les erreurs évidentes? Mais en l'absence des manuscrits originaux, comment départager les bévues typographiques de celles qui sont la résultante de l'inattention de l'auteur? Quant à certaines démonstrations mathématiques manifestement erronées, fallait-il les laisser telles quelles ou en donner la formulation correcte?

L'utilisation analogique des normes en cours dans l'édition des textes littéraires ne nous était ici d'aucun secours.

Il ne me restait alors qu'à reprendre le plan élaboré par Pasquale Boninsegni et Vladimiro Furlan, trouvé dans les archives de l'éditeur François Rouge et mis à ma disposition par Pierre Boven. Avec le seul soutien de Alain Dufour, éditeur, j'ai décidé alors de réaliser ce projet. L'obstination qui me poussait à prouver à tout prix que j'étais capable de faire seul ce que les autres considéraient impossible, que je pusse me passer de collaborateurs, des services d'un secrétariat et des aides techniques - cette obstination m'empêcha de voir clairement que le plan BoninsegniFurlan était incohérent, que Pareto avait donné son accord sans l'avoir véritablement examiné de près, et surtout que la répartition des écrits n'était guère conforme ni à l'ordre chronologique ni à l'ordre systématique. En effet, le plan prévoyait quinze volumes ainsi distribués: 1. Le Cours d'économie politique; 2 . Les écrits sur le marché financier italien; 3 . Les écrits autour de la courbe sur la distribution de la richesse; 4 . Les chroniques à propos du libre-échangisme, du protectionnisme et du socialisme; 5 . Les systèmes socialistes ; 6 . Les écrits sur les mythes et les idéologies politiques; Le Manuel d'économie politique; 8. Les articles de statistique et d'économie mathématique; 9. Tous les mémoires sur le marxisme et l'économie pure; 10. Les chroniques économico-sociales; 11. Le programme et le sommaire du cours de sociologie; 12. Le Traité de sociologie générale; 13. Les articles sur la transformation de la démocratie; 14. La liberté économique et les événements d'Italie; 15 . Le mythe vertuïste et la littérature immorale.

Tout le monde sait que le Cours... avait paru entre 1896-1897; les textes du $\mathrm{II}^{\mathrm{e}}$ volume entre 1891 et 1899 ; ceux du III $^{\text {e }}$ entre 1896 et 1897 et ceux du IV $^{\text {e }}$ entre 1887 et 1901. Les systèmes socialistes avaient paru en 1901-1902, le Manuel d'économie politique en 1909 , alors que les textes du VII ${ }^{\mathrm{e}}$ volume avaient paru entre 1891 et 1923 , du VIII ${ }^{\mathrm{e}}$ entre 1892 et 1901 , du IX ${ }^{\mathrm{e}}$ entre 1892 et 1919 , du X entre 1876 et 1921, du XI ${ }^{\mathrm{e}}$ en 1905. L'édition Boven du Traité... prévue pour le $\mathrm{XII}^{\mathrm{e}}$ volume avait paru en 1917-1919. Le XIII ${ }^{\mathrm{e}}$ volume contenant des articles 
imprimés pour la première fois en 1921, alors que le XIVe avait paru sous la forme actuelle en 1898. Au XV volume, enfin, était destiné Le mythe vertuïste..., mais dans son édition de 1911.

Dès la préparation pour l'impression du premier volume, j'ai mesuré aussitôt la fragilité, voire l'incohérence du plan Boninsegni-Furlan. En s'appuyant sur l'autorité de Guido Sensini, Bousquet souhaitait signaler toutes les erreurs et les coquilles d'imprimerie, mais en laissant le texte tel qu'il eût été établi par l'auteur. De son côté, Luigi Solari estimait qu'il fallait éliminer les bévues les plus évidentes et les coquilles et donner avant tout un texte qui fût aisément lisible par les économistes. Quant à moi, j'étais indécis sur la voie à suivre, pour toute une série de raisons. En voici un exemple. Dans le tome II du Cours..., § 724, il est écrit: «Si, dans un certain emploi, l'épargne reçoit un intérêt plus élevé que dans un certain autre, cela indique que le premier emploi est plus 'productif' que le second, et qu'il y a avantage pour la société à diminuer le premier emploi de l'épargne et des capitaux, et à augmenter le second». Ici, la bévue est évidente et indiscutable. Cependant, peut-on corriger sans autre par «augmenter le premier emploi de l'épargne et des capitaux, et diminuer le second »? La correction-rectification se serait imposée si Pareto n'avait pas utilisé dans d'autres écrits l'identique formulation. Renzo Fubini, le traducteur italien du Cours..., après avoir consulté Luigi Einaudi, décida de laisser le passage tel quel dans l'excellente version italienne qu'il nous a donnée. D'autres cas sont moins simples que celui que je viens de citer. Inadvertances, bévues, erreurs? Autre exemple, dans le tome I, $\S 83$, note 3 , du même Cours..., là où Pareto expose la relation entre le surplus du consommateur et l'ophélimité totale du consommateur d'après Alfred Marshall. M. Robin a démontré que l'équation est fausse.

En effet, si: $d V=\left(p_{b}-P_{b}\right) d \rho_{b}$

et que: $d \Phi=\left(1-\frac{P_{b}}{p_{b}} \varphi_{b}\left(\rho_{b}\right) d \rho_{b}=\frac{p_{b}-P_{b}}{p_{b}} \varphi_{b}\left(\rho_{b}\right) d \rho_{b}\right.$

on aura: $d V=\frac{p_{b}}{\varphi_{b}\left(\rho_{b}\right)}\left(\frac{p_{b} P_{b}}{p_{b}} \varphi_{b}\left(\rho_{b}\right) d \rho_{b}=\frac{p_{b}}{\varphi_{b}\left(\rho_{b}\right)} d \Phi \frac{1}{\varphi_{a}\left(\rho_{a}\right)} d \Phi\right.$

et non $\mathrm{dV}=\frac{\varphi_{\mathrm{b}}-\rho_{\mathrm{b}}}{\mathrm{p}_{\mathrm{b}}} \mathrm{d} \Phi=\rho_{\mathrm{a}}\left(\rho_{\mathrm{a})} \mathrm{d} \Phi\right.$, comme l'imprime Pareto.

Puisque Pareto veut montrer que les différentielles du surplus du consommateur et de l'ophélimité totale ne sont pas identiques mais proportionnelles lorsque l'ophélimité élémentaire de la marchandise qui sert de monnaie est constante, sa proposition n'en est pas moins démontrée. Il en est de même des équations dans le tome I, $\S 114$, note 1 . (Deux équations différentes portent le numéro (6) et d'après le texte ce serait plutôt l'équation (7), et non la (6) que l'on ne saurait accepter). Dans le tome II, $\S 698$, note 2 , les variations du prix et du coût de production sont représentés par une série de Fourier. Dès lors, il faut lire sin $t$ au lieu de sen $t$. Dans le tome II, $\S 958$, note 1 , l'équation 3 doit s'écrire: $\log \mathrm{N}=\log \mathrm{A}-\alpha \log (\mathrm{a}+\mathrm{x})$ et l'exposant $\alpha$ a été omis dans les équations (2 bis), (4) et (5). Dans le même tome II, § 974, note 1, il faut lire: 


$$
\frac{\delta \mathrm{r}_{\mathrm{b}}}{\delta \mathrm{p}_{\mathrm{a}}}=\mathrm{p}_{\mathrm{b}} \frac{\delta \mathrm{m}}{\delta \mathrm{p}_{\mathrm{a}}} \frac{1}{\varphi_{\mathrm{b}}^{\prime}} \text { et non } \frac{\delta \mathrm{r}_{\mathrm{b}}}{\delta \mathrm{p}_{\mathrm{b}}} \text {. }
$$

Dans les «additions » (tome II, p. 418), l'équation (5) doit s'écrire:

$$
\mathrm{g}=\mathrm{Hp}^{-8} \text { et non } \mathrm{y}=\mathrm{Hp}^{-8}
$$

Que fallait-il faire?

L'éditeur Droz trancha pour une solution médiane. En effet, il décida la reproduction du texte sans aucune modification et nous conseilla d'ajouter dans l'introduction une liste d'addenda et corrigenda.

Le volume IIe me posa d'autres problèmes. Presque toutes les données chiffrées étaient inexactes. Les totaux dans les tableaux étaient presque toujours faux. Corriger les additions et les pourcentages? Et si l'erreur dérivait d'une modification involontaire du typographe non décelée par l'auteur? Était-ce une modification dans la ligne ou dans la marge? Là où il était possible de remonter aux sources, la correction était facile à faire. Dans les autres cas, fallait-il laisser les choses en l'état? C'est cette dernière option que j'ai prise.

Le IIIe volume devait-il contenir les articles figurant dans le plan BoninsegniFurlan, ou bien tous les écrits de Pareto autour de la courbe de la distribution de la richesse? J'ai choisi la première position. Ainsi ne figurent pas dans ce volume les deux articles sur Les revenus des contribuables, parus dans la «Gazette de Lausanne» des 9 et 21 décembre 1896, ni non plus celui qui est sur la distribution de la richesse d'après le rôle de la taille à Paris, publié en juillet 1900.

Il serait trop long d'énumérer tous les problèmes que chacun des volumes m'a posés et les solutions, souvent assez bancales, que j'ai dû imaginer. Sur un point, hélas !, j'ai été très rigide: le respect absolu du plan Boninsegni-Furlan. Cette fidélité m'a posé de gros problèmes. Prenons le cas du Manuel d'économie politique, constituant le tome VII des Euvres complètes. La première édition, rédigée en italien, parut en 1906. Cette traduction française, revue par l'auteur mais faite par Alfred Bonnet, fut publiée en 1909. Un appendice (pp. 539-671) fut ajouté et inséré, sans modifications, dans la IIe édition italienne de 1909 du Manuale. La traduction de Bonnet est assez mauvaise. Pareto en était insatisfait mais avait consenti à la faire paraître. Que faire? Publier le Manuel ou le Manuale? Corriger le Manuel? Suivre l'exemple des traducteurs espagnol et anglais? mais était-ce le bon exemple? Cette exception au plan Boninsegni-Furlan est représentée par le XIe volume. Pour étoffer un peu le volume, j'ai ajouté en appendice le texte de Mon Journal que Tullio Bagiotti avant publié en phototypie en 1958 dans la collection de l'Université commerciale Bocconi de Milan. Fallait-il accoler un texte «scientifique» de 1905 à un écrit d'un genre «autobiographique» de 1918 ? En 1967, j'étais de l'avis que Mon Journal pouvait éclairer la démarche suivie par Pareto dans la construction des théories sociologiques. À présent, je ne le pense plus. Cette entorse au plan Boninsegni-Furlan est injustifiable. Seule la sociologie de la connaissance pourrait nous dire dans quelle mesure les obscurités, les ambiguïtés, voire les insuffisances des analyses de Pareto ont pu contribuer à faire de lui un auteur «maudit», et si ses apports isolés constituent une avancée décisive par rapport à d'autres travaux de son temps. 
La question du Traité..., constituant le XII ${ }^{\mathrm{e}}$ volume des CEuvres complètes, a été résolue grâce à l'intervention de Raymond Aron. L'éditeur Droz a longtemps hésité avant de s'engager dans la reproduction photomécanique de l'édition de 1917-1919. L'édition critique n'a paru qu'en 1988, en quatre volumes d'environ trois mille pages, mais seulement en langue italienne.

Le dernier volume, le XVe prévu par le plan Boninsegni-Furlan, était le Mythe vertuïste et la littérature immorale, mais dans l'édition française de 1911. Or l'édition italienne de 1914 est une véritable deuxième édition accrue et complétée. Après bien des tergiversations, j'ai pris la décision de re-imprimer l'édition de 1991 mais en y incorporant les ajouts, les compléments et les variations de l'édition de 1914, traduits en français par Micheline Tripet. Etait-ce une solution défendable sur le plan purement philologique? En tout cas, mes doutes sur la validité de cette solution se sont renforcés au fil des années.

Avec ce quinzième volume, j'aurais dû m'arrêter, mais la tentation de publier des articles qui avaient été écartés fut irrépressible. Et c'est ainsi que nacquît le XVI ${ }^{\mathrm{e}}$ volume intitulé Ecrits épars, contenant des textes parus entre 1888 et 1923 , sans liens logiques entre eux et pour lesquels une place appropriée aurait pu être trouvée dans les volumes III, IV, VI, VIII, IX, X, XI. Après la parution du XVI ${ }^{\mathrm{e}}$ volume, la diffusion des Euvres complètes commença à soulager un peu les investissements de l'éditeur. En outre, on me faisait observer de divers côtés que l'édition n'était pas complète malgré un affichage contraire sur les pages de titre. En effet, beaucoup d'articles manquaient et toutes les correspondances ne s'y trouvaient pas. Sans trop réfléchir à la question, j'ai alors réuni les articles politiques parus entre 1872 et 1923, dans les volumes XVII ${ }^{\mathrm{e}}$ et XVIII ${ }^{\mathrm{e}}$, et les articles sociologiques dits mineurs, parus de 1877 à 1923 dans le volume XXII ${ }^{\text {e }}$ La totalité de ces articles est en langue italienne. N'ayant pas pu les traduire en français, à l'exception de ceux qui sont contenus dans Faits et théories (volume $\mathrm{XXI}^{\mathrm{e}}$ ), tous ces volumes font une entorse au principe de donner uniquement les textes rédigés en français, principe formulé dans le plan Boninsegni-Furlan.

En outre, un petit nombre de ces articles, avec des variations minimes, parfois même avec quelques changements, avaient paru dans l'un ou l'autre des premiers quinze volumes des CEuvres complètes. Ces articles ont tout de même été publiés intégralement avec en note l'indication des versions précédentes. La solution la plus convenable eut été de faire état, dans les notes de la première publication, des variantes et des autres éditions. Cependant, l'économie générale du plan, tout au moins au commencement, s'y opposait.

Il en a été de même pour les écrits de jeunesse (volume $X X V^{\mathrm{e}}$ ), pour les écrits d'économie politique pure (volume $\mathrm{XXVI}^{\mathrm{e}}$ ) et pour le premier cours d'économie politique appliquée professé à l'Université de Lausanne (volume XXIVe).

Tous les écrits retrouvés après la publication de cette seconde série de volumes sont aujourd'hui regroupés dans le volume XXIX ${ }^{\mathrm{e}}$. Depuis, d'autres écrits ont été découverts. Quelques-uns ont été placés en appendice aux nouvelles éditions de certains volumes épuisés des CEuvres complètes. C'est, par exemple, le cas de deux inédits publiés en appendice de la deuxième édition du volume VIII D'autres textes, par exemple la première version du cours d'économie politique pure donné à Lausanne en 1893-1894, restent encore inédits.

Les actes du Jubilé Pareto de 1917 composent le volume XXe ${ }^{\mathrm{e}}$, dans lequel se trouvent également deux bibliographies: une des écrits de Pareto (dont une 
version plus complète est présente aussi dans le volume $\mathrm{XXX}^{\mathrm{e}}$ ) et une autre des écrits sur Pareto jusqu'à 1975. Pour la période postérieure, la bibliographie se trouve dans l'édition critique italienne du Traité de sociologie générale.

La correspondance compose les volumes XIX ${ }^{\mathrm{e}}$ (paru en 1975), XXIII (paru en 1981) XXVII et XXVIII ${ }^{e}$ (parus en 1984), ainsi que le volume $X^{\text {XX }}{ }^{\mathrm{e}}$ publié en 1989, le volume XXXI paru en 2001 et clôturant l'entreprise. Tous ces volumes de correspondance ayant été composés à des moments différents et sur la base des lettres alors disponibles, ils se chevauchent chronologiquement et cela complique la consultation et l'étude de certaines questions traitées dans différentes lettres de la même année, mais placées en des volumes différents. Les tables de concordance et de chronologie essaient de pallier à ces difficultés, mais elle ne les éliminent pas intégralement.

Sans aides privées, sans subventions officielles, sans aucune assistance ou collaboration, préparant souvent la copie en dehors des heures de bureau, soutenu uniquement pas un éditeur dont le désintéressement économique continue à me paraître extraordinaire, j'ai publié une trentaine de volumes, tantôt en reproduisant tels quels d'anciens textes, tantôt en les composant typographiquement de bout en bout. Dans les premières éditions de ces volumes, il n'y a pas toujours d'index. Dans les deuxièmes, on a commencé à éliminer cette grave lacune.

Bien entendu, la structure fondamentale de cette édition des écrits de Pareto n'est pas satisfaisante. Les règles philologiques n'y sont pas strictement respectées, mais aurais-je pu faire autrement dans la situation où je me trouvais? Avec d'autres contraintes, aurais-je pu, comme je l'ai fait, publier à moi seul la presque totalité des écrits de Pareto en vingt-six ans, et le faire en parallèle à mes activités professionnelles principales?

Répondre à ces questions ne me paraît guère utile. Mieux vaut, me semble-t-il, essayer de tirer quelques enseignements de cette expérience personnelle. La seule chose à laquelle j'aurais dû être plus attentif, c'est de ne pas me mettre à dos tant de collègues importants. J'aurais dû éviter les polémiques et les bagarres intellectuelles. Je reconnais aujourd'hui que le travail d'édition est un travail compliqué, difficile, qu'il est opportun de mener en équipe. L'édition de textes, peu valorisée chez les sociologues et les économistes, devrait avoir une plus grande légitimité et une meilleure reconnaissance académique. Sans des éditions fiables, il est impossible de connaître et reconnaître les modes de production des théories, la façon dont la science normale se constitue et se diffuse, les mécanismes produisant les révolutions scientifiques, les procédures permettant à telle théorie de prévoir des faits nouveaux et à telle autre d'expliquer certaines anomalies; de saisir, aussi, pourquoi la simplicité, l'élégance, la généralité d'une théorie lui confèrent presque toujours une extrême fécondité. En science sociale, à l'heure actuelle, il n'existe aucun système cohérent et intégré pour l'édition des textes fondamentaux de nos classiques et de nos auteurs les plus marquants. Nos sociétés savantes devraient à l'avenir favoriser la formalisation de certaines pratiques actuelles et fixer les normes standard pour la publication de nos textes scientifiques. Nous ne pouvons pas transposer automatiquement les règles gouvernant l'édition des textes littéraires dans nos domaines; nous devons en établir de nouvelles pour des auteurs dont le souci majeur reste la construction de théories et l'élaboration de doctrines.

J'admets que mon édition est insatisfaisante, voire carentielle. Cependant le fait de pouvoir remettre en circulation, même au moyen d'anastatiques, les textes 
originaux que tout le monde citait mais que personne n'avait pu lire jusqu'alors, puisqu'ils étaient introuvables en librairie depuis longtemps, me parut une étape importante pour soustraire les discussions aux seuls initiés et les rendre ainsi accessibles à tous. Pourtant, si mon intention était toujours de contribuer à développer à travers Pareto l'histoire des sciences sociales, à réévaluer le positivisme et les courants empiriques, bref, à faire en sorte que l'histoire de la culture ne soit pas toujours limitée à l'histoire littéraire et philosophique, deux autres directions commençaient à me préoccuper: la première est celle qui vise à étudier la pensée économique de Pareto comme idéologie de l'industrialisation et de la modernisation, relativement au climat socio-économique dans lequel les institutions de la démocratie libérale et du mouvement ouvrier se sont développées ou péri avec l'avènement du fascisme; la seconde est celle que Raymond Aron a nommée la «fin des idéologies», et qui a été brillamment illustrée en Italie par Norberto Bobbio sous le concept de «critique des idéologies».

Nul doute que les revues critiques des publications de 1966 à 1973 laissent transparaître ces deux nouvelles intentions, de même qu'une croyance personnelle, à savoir que le réalisme désenchanté du vieux sociologue, dégagé de toutes formes d'implications pratico-politiques, peut contribuer à nous débarrasser des illusions et des mythes sociaux, à voir plus clair dans nos espérances et dans nos engagements, bref, à vivre plus sagement, même dans les moments idéologiquement rudes et difficiles, sans toutefois renoncer à nos sagesses personnelles, mais sans non plus les élever au rang de jugements absolus. En d'autres termes, le réalisme de Pareto pourrait fournir un vaccin contre les injustices, contre la bêtise humaine, contre le découragement.

Que mes travaux parétiens soient parvenus à réaliser les buts que je me fixais, je n'en suis pas du tout convaincu. Pareto continue à ne pas avoir une place canonique dans l'histoire des sciences sociales. Pourquoi?

L'histoire de la sociologie n'a ni la tradition ni le patrimoine de connaissances accumulés par l'histoire de la philosophie et pourtant elle peut déjà compter sur un système de références solides et relativement consensuelles. L'accord sur les critères nécessaires pour ranger un sociologue parmi les classiques de la discipline en est un bon spécimen.

Ces critères sont au nombre de trois. Un sociologue est réputé classique: 1 . si ses travaux fournissent une interprétation cohérente des problèmes de la société dans laquelle il a vécu; 2 . si la lecture de son œuvre est actuelle et reste à la base de nouvelles élaborations théoriques; 3. si, enfin, il a élaboré des théories bien formées ou construit des modèles dont on se sert encore pour expliquer la réalité sociale, fût-elle différente de celle les ayant engendrés ou à laquelle ces théories et ces modèles ont été primitivement appliqués.

Presque tous les sociologues estiment que les travaux de Marx, de Durkheim, de Weber, mais aussi de Tocqueville, de Simmel et de quelques autres, possèdent les trois caractéristiques mentionnées, d'où leur place canonique parmi les classiques de la discipline. Par contre, la place de Vilfredo Pareto est rudement contestée et soulève une multitude de réserves importantes. En France, par exemple, Raymond Aron a souvent écrit que Pareto «ne jouira jamais, sur cette terre, d'une reconnaissance un unanime et pacifique», Raymond Boudon est convaincu que le purgatoire de la sociologie parétienne «risque de se prolonger encore un peu», tandis que Henri Mendras et beaucoup d'autres considèrent que 
le savant italien ne peut être rangé parmi nos pères fondateurs car son œuvre n'offre aucune aide aux chercheurs contemporains.

En vérité, les raisons de cet état de fait sont singulières. Les critères employés pour qualifier un sociologue de classique pourraient s'appliquer sans autre à Pareto: ses analyses de la société européenne de 1870 à 1923 sont utilisées avec profit même par les historiens, les innombrables lectures de ses travaux ont donné naissance à une véritable herméneutique, à des reformulations théoriques importantes et ses théories et ses modèles sont utilisés par des chercheurs de terrain. «Middletown III Research », à laquelle Theodore Caplow a activement participé, et les innombrables enquêtes sur les élites dont John Scott a tracé l'historique dans trois gros volumes parus en 1990, en fournissent quelques aperçus probants.

Pourquoi alors cette étrange, pour ne pas dire bizarre destinée?

Les réponses données jusqu'ici à cette question ne paraissent pas très congruentes. Pareto n'est pas le seul sociologue «outsider» à débattre avec sympathie de la force et de la violence, à écrire des livres monstrueux, hermétiques, mélangeant les genres, à narguer tout le monde, à braver les règles, les conventions, les coutumes de la communauté scientifique d'appartenance, à voltiger d'une discipline à l'autre, à avoir quelques faiblesses pour les régimes politiques autoritaires, à afficher crûment son cynisme et son pessimisme. Par contre, il est assurément le seul, dans l'histoire de la discipline, à rejeter, sans ménagement, le patrimoine commun, le positivisme, l'approche utilitariste de l'action, le schéma analytique «moyens-fins ». Il est encore le seul à récuser l'intangibilité des notions d'objectivité, de progrès, de rationalité du monde historicosocial, en somme à se situer à l'opposé des options théoriques fondatrices de la sociologie, à proposer, avec une conceptualisation ésotérique et dans un langage sibyllin, des approches analytiques très problématiques, à se placer à contrecourant de la lettre et de l'esprit des paradigmes communs, à forclore de la vie ordinaire l'universalité méthodologique du principe de rationalité, là où la foi, les conflits et la communication persuasive sont hégémoniques.

L'écart entre les conceptualisations sociologiques de Pareto et celles des principales tendances de la théorie sociale de notre siècle est, sans aucun doute, considérable. Alors même que le socle de la sociologie est le positivisme, Pareto en parle comme s'il s'agissait d'une croyance métaphysique, d'un dogmatisme. d'un mélange factice d'intérêts cognitifs et d'utilités sociales, d'être et de devoir être, de théorie et de pratique, de science et de programmes sociaux. Comte, Spencer, Durkheim, et tant d'autres, sont qualifiés de fidèles de la religion humanitaire; leurs prétentions de pratiquer une science sociale réformiste taxée d'incongruités fallacieuses.

À l'option méthodologique du positivisme de fonder la discipline sur les conduites observables, commensurables, calculables, Pareto oppose un cadre d'interprétation du réel, disons une sorte d'empirisme logique privilégiant les habitudes langagières, les raisonnements, les représentations, la logique brute de la subjectivité irréductible au calcul, empirisme logique avant la lettre et qui différencie le langage naturel, dénommé aussi vulgaire, celui des croyances représentées, des décisions, des actions de la vie quotidienne, du langage artificiel de la science. À cette dernière, il assigne la tâche d'analyser les motivations irréfléchies de la vie sociale, les justifications données, les significations attribuées aux actions historiquement déterminées, de dévoiler les systèmes de représentations 
orientant et organisant nos relations au monde, avec d'autres mots: les mécanismes composites producteurs des univers symboliques de la société. Les représentations élaborées pour justifier et puis faire valoir une croyance ou une action ont des visées pratiques, sont étrangères à la science logico-expérimentale. La logique de l'action n'est pas la même que celle de la cognition scientifique.

L'ordre social n'est pas assuré par des systèmes normatifs mais par des espèces d'archétypes, mieux: des radicaux aux préfixes et suffixes multiples, constituant des totalités signifiantes en rapport avec l'action. Ils structurent les liens sociaux, déterminent les capacités d'actions des acteurs, cristallisent les actions individuelles en unités collectives, font des individus isolés une totalité, les constituent en une société. Ils forment des savoirs pratiques socialement élaborés et partagés.

De la description du phénomène concret, aux formes souvent changeantes, description fondée exclusivement sur l'observation et l'expérience (cette dernière n'étant qu'une collection d'observations passées), l'analyse abstrait les aspects que la théorie et les hypothèses ont postulé comme étant répétitifs et/ou en connexion avec des aspects analogues et similaires d'autres phénomènes. Les variables construites ainsi que leurs interdépendances, composent un corpus de lois, dites aussi « uniformités », et qualifiées tantôt de virtuelles et tantôt de conditionnelles (si...alors). La correspondance entre l'énoncé linguistique et l'observation empirique détermine la vérité de l'utilité, le vrai du faux, le logique du non-logique. Dans le Traité..., il est écrit que: «[...] la science expérimentale remonte des cas concrets, non pas à des principes absolus, qui pour elle n'existent pas, mais seulement à des principes généraux que l'on fait ensuite dépendre d'autres $[\ldots] \gg(\S 22)$. Cette science conçoit les lois en tant qu'uniformités observationnelles, contingentes, probables, approximatives, limitées contextuellement et temporellement. Ces uniformités sont explicitement considérées comme des conventions. Dans cette approche, «les théories scientifiques sont des simples hypothèses, qui vivent tant qu'elles sont d'accord avec les faits, et qui meurent et disparaissent quand des nouvelles études détruisent cet accord $»(\$ 52)$. La distinction-opposition postulée entre savoirs cognitifs et savoirs pratiques, comporte que «la logique cherche pourquoi un raisonnement est erroné, la sociologie pourquoi il obtient un consentement fréquent » (\$1411).

Dès lors la tâche du sociologue consiste à mettre en évidence, au moyen de modèles, de fictions conceptuelles, la structure constante, non consciente, intemporelle, ainsi que les modalités spécifiques, variables, des cristallisations des interprétations non empiriques, les contenus et les formes de pensée du sens commun, tout ce qui fait sens, même si celui-ci n'est pas immédiatement décodable.

Dans cette perspective, l'objet d'étude du sociologue, ce sont les raisonnements, les délibérations, les argumentations, la logique des sujets, l'objectivisation du fonctionnement subjectif, c'est-à-dire ces savoirs déclaratifs et procéduraux qui anticipent et prescrivent les actions des acteurs sociaux.

La thèse selon laquelle la réalité sociale est formée d'une partie constante, non consciente, saisissable pourtant au travers des interprétations variables, conscientes, sédimentées en un système symbolique, qu'il y a une logique de la démonstration et une logique de l'argumentation, aux finalités distinctes et discordantes, une telle thèse est fortement décalée par rapport à la culture sociologique 
standard de la première moitié de notre siècle. En porte à faux indéniable lorsqu'elle célèbre les vertus de la démarcation entre le contexte de l'invention des hypothèses et le contexte du contrôle des énoncés. L'idée qu'on puisse faire feu de tout bois au moment de l'invention et que la méthode logico-expérimentale s'applique à la seule démonstration, cette idée était d'autant plus irrecevable que son auteur qualifiait en plus les preuves inductives d'intrinsèquement contingentes, dépassables, donc relatives à un état donné de la recherche.

L'incompatibilité des constructions théoriques parétiennes avec la sociologie standard est encore plus patente à propos de la raison, du rationalisme et de la rationalité. Pour tous les sociologues, l'action rationnelle était et est celle de l'acteur social qui organise son agir de façon consciente, continue, par rapport à un ou à des systèmes de significations. L'action est rationnelle si l'acteur, pour réaliser ses objectifs, se réfère constamment et méthodiquement à un ou à des systèmes de significations irréductibles à une utilité empirique contingente. Pour la plupart des sociologues, les fins sont de données, en tout cas toujours repérables. Pour Pareto les fins sont celles qui se réalisent au point d'équilibre, donc elles ne sont aucunement réductibles à la rationalité formelle des langages scientifiques. A l'exclusion de certaines actions où le rapport moyen-fin est assuré, toutes les autres sont étrangères à ce rapport. Pour cette raison, elles sont non logiques, pas illogiques. Ce fait n'étant pas la résultante d'une erreur, l'analyste doit l'interpréter et il peut l'interpréter seulement en termes de langage naturel producteur de sens, d'argumentations sollicitant des adhésions et irréductibles à la rationalité des langages formels. Ainsi, la rationalité de la science est opposée derechef à celles de l'action et de la décision, la connaissance objective des savants à celle subjective des acteurs, les explications par les raisons conscientes, ou susceptibles de le devenir, à celles se trouvant à la base de l'agir individuel et social.

La tendance des hommes à la ratiocination, à argumenter pour rendre plausibles et acceptables leurs comportements et leurs croyances, cette tendance n'est ni un instinct ni une inclination; elle est le fondement même de l'existence sociale. Cette ratiocination réalise et garantit l'intersubjectivité des interprétations que les acteurs sociaux donnent des contextes et des situations dans lesquels ils agissent. Pour rendre compte de ces mécanismes producteurs des ententes tacites, à défaut desquelles les échanges sociaux seraient irréalisables, Pareto analyse les discours des acteurs sociaux, la mise en forme des preuves verbales, les structures enthymématique des raisonnements, les différents types d'ambiguïtés, les paralogismes et les effets disparates qui en résultent. Pour la première fois dans l'histoire de la sociologie, le langage est traité en tant que réquisit principal de la socialité, antérieur à toutes les formes de rationalité logico-expérimentale, en tant qu'instrument, d'une part, de communication avec autrui, d'échanges symboliques et, d'autre part, en tant qu'outil pour la construction, au moyen de classes-objets discursives, des notions qui structurent les univers symboliques, qui rendent plausible la justification des croyances, des décisions et des actions.

En bref, les résidus, ces sortes de préconstruit, à la fois contenu et connaissance du sens commun, source de la socialité - les résidus, par l'entremise du langage, produisent les ententes tacites qui charpentent le système social, l'agencement des parties en un tout; ils octroient aux acteurs une «logique» toute faite, donnée d'avance, et au même moment ils exercent, pour ainsi dire, des fonctions d'identification, de représentation et de nomenclature. Leur composition s'avère 
être le prolongement d'une espèce d'organisation spontanée, équilibrée, autorégulée, des actions de classement, de sériation, de persuasion.

Sont-ils des formes perceptuelles a priori semblables à celles qui sont ressuscitées par des néo-innéistes tels que Noam Chomsky ou Jerry Fodor? Sont-ils proches des «themata» de Gerald Holton, des jugements enracinés dans la psychologie? En d'autres termes, sont-ils les résidus des jugements, issus ni de l'observation objective ni d'une activité de type logique, innés ou liés au langage (mais comment?) ou enfin sont-ils les fruits d'une stratégie sélective de la nature, pour ainsi dire, bioculturelle évolutive, que les spécialistes de l'évolution du comportement nomment biogramme? Avec d'autres mots, le substrat génétique serait-il représenté par certaines prédispositions du comportement qui se manifestent principalement dans ces universaux culturels que Pareto appelle les résidus? Est-ce que les résidus, les dérivations, les intérêts ainsi que l'hétérogénéité et la circulation sociale sont des manifestations plus ou moins directes de facteurs phylogénétiques atteints par inférence? Sont-ils des moyens pour forcer l'individu à rechercher sont utilité maximale en tant qu'individus à maximaliser sa capacité d'adaptation au groupe?

Ces questions sont restées jusqu'ici sans réponses. En effet, tous ceux qui ont étudié les chapitres VI, VII et VIII du Traité... se bornent à dire que les résidus sont un embrouillamini typologique très énigmatique. Tous sont de l'avis, à deux ou trois exceptions près, que cette théorie des résidus est mal formée, qu'il s'agit d'une bien mauvaise typologie, qu'elle est tout au plus un schéma explicatif affectiviste, une doctrine émotiviste et anti-intellectualiste, aboutissant, par conséquent, au subjectivisme et à l'irrationalisme. Pour ma part, je crois qu'une lecture plus attentive à l'esprit qu'à la lettre du Traité... obligerait à nuancer ces propos.

Pareto dit explicitement ( $\$ 875$ et $§ 1689)$ que les résidus ne sont pas des instincts ni même des sentiments, qu'ils sont à la fois la source et l'expression constantes des raisonnements, des représentations qu'il dénomme «dérivées» et «dérivations». Les résidus se transforment lentement, ils constituent un cadre d'interprétation fournissant la signifiance stable des comportements, ils restructurent et unifient les fins mais ils demeurent irréductibles à la rationalité formelle. Ils rendent possibles l'intersubjectivité et l'intelligibilité, en somme l'existence de la société en tant que totalité intégrée, bien que «les notions que les différents individus ont au sujet de ce qui est bien pour eux-mêmes ou pour autrui [soient] essentiellement hétérogènes, et qu'il n'y [ait aucun] moyen de les réduire à l'unité»".

Étant donné qu'«une société déterminée exclusivement par la 'raison' n'existe pas et ne peut pas exister « et que les fins sont indéterminées et indéterminables scientifiquement, seuls les résidus peuvent légitimer les actions individuelles et collectives au moyen de significations universelles octroyées au travers du langage. Par l'entremise du langage », « on parvient jusqu'aux sentiments des hommes» et, par celui-ci «on peut en conséquence modifier leur activité » (§1403). Le langage, «ayant une existence indépendante des choses, peut paraître exercer sur elles une influence plus ou moins grande, et en exerce certainement une sur les théories que l'on fait au sujet de ces choses». En plus, «le langage vulgaire est habituellement synthétique, lorsqu'on en use, [et] tient compte, bien que rarement et mal il soit, de la mutuelle dépendance des phénomènes ». 
En d'autres termes, les résidus transcendent, implicitement et explicitement, l'expérience et la logique, se situent au-delà des langages scientifiques, et donnent une grande autonomie, une certaine prépondérance, à la langue naturelle. Leur rationalité basique assure le contrôle de la vie en tant que tout, la docilité aux principes de légitimité, la conformité des comportements individuels aux projets d'ordre supérieur.

Une analyse approfondie des résidus révèle que Pareto a voulu construire une typologie des processus sociaux macrosociologiques à partir de la dichotomie universalisme/particularisme, où l'universalisme est le code et le particularisme est le contexte. Pour y parvenir, il fait recours à des très nombreuses descriptions de contextes tandis que l'usage constant de tropes, par métaphores, métonymies et synecdoques, l'aide à mettre en évidence les similarités, les analogies ou bien les oppositions de et entre les différents contextes décrits. La dichotomie universalisme/particularisme est généralisante pour les résidus de la classe 1 (Instinct des combinaisons) et de la classe 2 (Persistance des agrégats). Les classes 3 (Besoin de manifester ses sentiments par des actes extérieurs), 4 (Résidus en rapport avec la sociabilité), 5 (Intégrité de l'individu et de ses dépendances) et 6 (Résidus sexuels) sont des spécifications et des particularisations des deux premières classes, sous lesquelles, par ailleurs, il est aisé de les subsumer.

Les quatre classes de dérivées et des dérivations (Affirmation, Autorité, Accord avec des sentiments ou avec des principes, Preuves verbales) rendent l'aperception des résidus possible en les exprimant discursivement. Elles n'ont aucune autonomie ni même de logique appropriée à leur rhétorique pratique; elles sont inaptes à transformer les assertions en propositions contrôlables. Derivées et dérivations restent surtout les révélatrices des résidus, des contenus, des sujets et de raisonnements de la logique naturelle dans laquelle chaque objet et chaque prédicat peuvent s'enrichir progressivement d'informations ou de connotations rapportées après coup. L'étude des résidus en tant qu'éléments constants de tous les systèmes symboliques pousse Pareto vers l'analyse des structures latentes de l'agir social alors que celle des dérivées et des dérivations vers la description suggestive et variée de leurs manifestations historiques. Certes, les résidus varient au cours des étapes du développement, cependant les variations se compensent. Ainsi, les classes restent toujours constantes et en dépit de leur hétérogénéité, elles possèdent les éléments indispensables pour assurer la constance de l'ensemble malgré les variations de détail.

Entre la formulation de généralisations au moyen de couples philosophiques apparence/réalité, fond/forme, et l'observation socio-historique des phénomènes, le rapport reste fictivement inductif. Rien n'est dit sur la structure du raisonnement, rien non plus sur la fonction des symboles ni pourquoi ces rationalisations contribuent à maintenir l'équilibre social. Pareto se borne à noter dans le $\S 1403$ : «En somme, les dérivations constituent les matériaux employés par tout le monde. Mais les auteurs précités donnent aux dérivations une valeur intrinsèque, et les considèrent comme agissant directement dans la détermination de l'équilibre social, tandis que nous leur donnons uniquement la valeur de manifestations et d'indices d'autres forces, qui sont celles qui agissent en réalité dans la détermination de l'équilibre social.»

Un tel axiome se trouve également à la base de la théorie du système social, théorie construite à partir des relations d'interdépendances des résidus, des déri- 
vations, des intérêts et de l'hétérogénéité et circulation sociales. Pareto opère ici de la même manière que pour le rapport résidu/dérivations. Par l'abstraction analytique, il individualise les variables et puis il recherche les uniformités caractérisant leurs interdépendances simultanées. L'équilibre n'est que l'observation de «l'état auquel nous voulons considérer le système social, dont la forme change continuellement. L'état réel, statique ou dynamique du système est déterminé par ses conditions ». Une quelconque modification dans sa forme produira aussitôt une réaction; «elle tendra à ramener la forme changeante à son état primitif, modifié par le changement réel. S'il n'en était pas ainsi, cette forme et ces changements ne seraient pas déterminés, mais demeureraient arbitraires » (§ 2068). En ajoutant qu'il en va de même pour les changements accidentels (guerres, épidémies, inondations, tremblements de terre et autres calamités), Pareto souligne avec vigueur que l'état d'équilibre n'est que la réalité observée selon les canons de la méthode logico-expérimentale et qu'il ne faut pas le confondre ni avec la causalité, ni avec l'incertitude, ni même avec l'imprévisibilité. C'est l'état d'un phénomène déterminé par les conditions qui l'ont engendré, c'est en bref, un processus de changement non casuel. Le point où la régulation atteint un état d'équilibre est défini par le moyen d'un ensemble d'égalités qui coïncident momentanément avec un système d'opérations réversibles. Ici aussi le système se base sur la substitution des égalités et sur l'entière conservation du tout. Or, précisément pour cette raison, Pareto doit postuler la simultanéité des actions et des effets, la réciprocité et la réversibilité des liens entre tous les facteurs de l'équilibre, et même la continuité des grandeurs et l'unicité des solutions. La théorie des cycles de mutuelle dépendance lui permet ensuite d'analyser, par éléments successifs, les effets des interdépendances de toutes les variables, mais toujours à un niveau de très grande généralité. Le système n'a ni des fins ni des objectifs extérieurs à lui et la notion de communication lui est étrangère. Toutes les perturbations de l'équilibre doivent nécessairement provenir du monde extra social. Dès lors, les changements sont impensables et les déséquilibres impliquent forcément leur retour automatique à l'équilibre. Pour cette raison, il est inévitable que les phénomènes sociaux aient une forme ondulée et que leurs oscillations soient «le résultat de l'antagonisme de deux forces opposées [distinctes et de sens différent]: la correspondance des dérivations à la réalité et leur utilité sociale». Puisqu' «il demeure, d'une part, sinon impossible, au moins malaisé de se soustraire entièrement à la réalité, et, d'autre part, de négliger entièrement l'utilité sociale, il en résulte nécessairement que dans tout ce qui se rapporte à l'organisation sociale, la théorie oscille comme un pendule, tantôt d'un côté, tantôt de l'autre».

Étant donné que le système social est un système des relations entre quatre variables et que tout changement dans la structure des interdépendances de ces mêmes variables, à défaut d'un retour à l'équilibre, du rétablissement du cycle de mutuelle dépendance, comporte inévitablement un changement de tout le système, Pareto ne nous donne pas une théorie de l'équilibre générale de la société mais seulement la théorie d'un système empiriquement déterminé, théorie inappropriée à expliquer la transition d'un système à l'autre, à rendre compte du pourquoi des déséquilibres. La cause d'un cycle de mutuelle dépendance est le cycle antécédent ainsi qu'il écrit dans le § 2338: «En réalité, les oscillations des diverses parties du phénomène social sont en rapport de mutuelle dépendance, à l'égal de ces mêmes parties. Si l'on tient à se servir du terme fallacieux de cause, 
on peut dire que la période descendante est la cause de la période ascendante qui la suit, et vice versa; donc, en général: que les différentes périodes sont seulement des manifestations d'un seul et unique état des choses, et que l'observation nous les montre en succédant les unes aux autres, de telle sorte que suivre cette succession est une uniformité expérimentale.» La théorie de l'équilibre générale ne nous dit jamais qu'est-ce qui est utile à la société et si cette utilité pourrait être établie par quelqu'un et à quelles conditions. Or, une société doit en permanence faire des choix et elle peut les faire dans la mesure où elle dispose de critères pour ordonner les préférences, pour déterminer et fixer les fins, pour réaliser tel objectif plutôt que tel autre. Ces critères sont externes à la notion d'équilibre, donc indéductibles. Dans l'impossibilité d'établir quel est le moyen approprié à une fin, de repérer la fin pour laquelle l'action est entreprise, d'homogénéiser les critères à la base des délibérations, des actions en vue de l'obtention d'une utilité, en bref, de connaître les fins, Pareto ne nous laisse aucune indication claire sur la façon de concilier la rationalité de l'action avec la rationalité épistémique, sur le comment insérer la rationalité épistémique dans l'interprétation empirique des actions historicosociales. La plupart des interprètes ont essayé de contourner ces difficultés, d'éliminer les apories en réduisant la sociologie générale de Pareto à une sociologie des idéologies, à une sociologie politique, à une sociologie des argumentations impures et imparfaites, à une sociologie de l'action historique et de la rhétorique politique.

Ces lectures sont assurément méritoires, certaines magistrales, cependant, elles négligent le projet principal de Pareto: celui de participer à l'élaboration d'une sociologie générale. Dans cette perspective-là, il est utile de relire aujourd'hui Pareto. La situation actuelle du débat intellectuel en sociologie nous permet de le lire autrement que dans le passé récent et surtout de l'interpréter à la lumière des acquis théoriques que nous avons accumulés au cours de la deuxième moitié du siècle révolu.

Quelques mots me suffiront pour dire pourquoi il est souhaitable que Pareto retourne parmi nous et trouve enfin dans l'histoire de la sociologie une place adéquate avec l'originalité de son œuvre. Il nous propose d'élucider le mystère des logiques inhérentes à la vie sociale, de découvrir comment elles opèrent, de quelle façon elles se représentent, pourquoi elles argumentent et peuvent faire l'économie de la démonstration. Il nous invite à réfléchir sur un problème central de notre modernité, celui de la raison, des raisons, des rationalités, des raisonnements. Il nous dit que la sociologie peut être une science, qu'elle peut rendre compte scientifiquement des savoirs de la vie pratique construits selon des règles différentes de celles de la logique inductive et déductive.

Si nous sommes convaincus que la sociologie n'a pas la mission de trouver des solutions à tous nos problèmes sociaux, si nous sommes convaincus que la sociologie est un moyen pour rendre les relations sociales intelligibles et si nous sommes aussi convaincus que la communication scientifique doit montrer comment l'homme croit, agit et produit pour vivre en société, alors l'œuvre de Pareto doit revenir parmi nous. Car elle nous aide à voir comment et pourquoi nous produisons certains savoirs, comment nous les utilisons pour vivifier nos espoirs, nos projets, nos décisions, nos choix, nos actions. Elle nous montre également leurs limites et leurs pauvretés. Produits par des normes particulières, profitables pour notre vie, ces savoirs ne sont ni éternels ni absolus. Des sagesses 
indispensables que la sociologie tend à rendre intelligible, intelligibilité toutefois historiquement déterminée. L'œuvre de Pareto peut nous aider à élaborer une sociologie scientifique, une sociologie critique des formes de production des savoirs et des connaissances, à comprendre pourquoi l'étude sociologique de la société est un moyen extraordinairement efficace pour rendre compte de l'agir social.

Pour toutes ces raisons, il faut aujourd'hui recommencer à lire Pareto et lui donner une place honorable dans l'histoire des sciences sociales ${ }^{1}$.

Chêne-Bourg, Genève.

Parmi les contributions récentes allant dans cette direction, il faut signaler Economia, Sociologia e Politica nell'opera di Vilfredo Pareto. A cura di C. Malandrino e R. Marchionatti, Firenze, Olschki, 2000, ainsi que Vilfredo Pareto (1848-1923). L'uomo e lo scienziato. A cura di G. Manca. Prefazione di A. Fazio. Con una Appendice di 63 lettere inedite del Fondo Vilfredo Pareto della Banca Popolare di Sondrio, Milano, Scheiwiller, 2002. 